Clinical Nutrition 2020, 39(7), pp. 2001-2013

\title{
Weight-related stigma and psychological distress: A systematic review and meta-analysis
}

\author{
Zainab Alimoradi ${ }^{\mathrm{a}}$ Farzaneh Golboni ${ }^{\mathrm{a}}$ Mark D. Griffiths ${ }^{\mathrm{b}}$ Anders Broström ${ }^{\mathrm{c}}$ Chung-YingLin ${ }^{\mathrm{d}}$ \\ Amir H.Pakpour ${ }^{\text {ac }}$
}

Social Determinants of Health Research Center, Research Institute for Prevention of

b

Non-Communicable Diseases, Qazvin University of Medical Sciences, Qazvin, Iran

International Gaming Research Unit, Psychology Department, Nottingham Trent

University, United Kingdom

Department of Nursing, School of Health and Welfare, Jönköping University, Sweden

Department of Rehabilitation Sciences, The Hong Kong Polytechnic University, Hung Hom, Hong Kong

\section{Summary}

\section{Background \& aims}

Individuals who are overweight or who have obesity are likely to perceive or experience unfriendly treatment (i.e., weight-related perceived stigma) from different sources such as work colleagues because of the stigma towards excess weight. People who are overweight may accept such stigma and devalue themselves (i.e., weight-related self-stigma).

\section{Methods}

A systematic review and meta-analysis was conducted to examine the relationship between weight stigma (including weight-related self-stigma and weight-related perceived stigma) and psychological distress (including depression and anxiety) using random-effects meta-analyses. Utilizing five academic databases (PubMed, Scopus, WOS, Embase and ProQuest) and keywords related to weight stigma and psychological distress, empirical studies focusing on the association between weight stigma and psychological distress were selected. The timeline for the searched papers was from the inception of each database to the end of August 2019.

\section{Results}

Eligible studies ( $\mathrm{N}=30 ; 25$ on weight-related self-stigma and eight on weight-related perceived stigma) were analyzed with a total of 9345 participants experiencing weight-related self-stigma, and 15,496 experiencing weight-related perceived stigma. The pooled associations were moderate between weight-related self-stigma and psychological distress (corrected Fisher's $Z=0.40$ for depression; 0.36 for anxiety) and between perceived stigma and depression (Fisher's $Z=0.44$ ).

\section{Conclusions}

Results of the meta-analysis demonstrated that weight stigma is associated with psychological distress. The comprehensive search of the literature and rigorous methodology employed are the two major strengths in the present study. Because self-stigma and perceived stigma are different concepts, their associations with psychological distress should not be merged together. 
Clinical Nutrition 2020, 39(7), pp. 2001-2013

\section{Keywords}

Weight-related stigma

Psychological distress

Systematic review

Obesity 